\title{
Astashine Capsules: A Natural Antioxidant, Anti-inflammatory Helps in Maintaining Lung Health and Minimizing SARS-CoV-2 effects in Covid-19 Infections
}

Govind Shukla, Uddhav L Kanade, Monica Yadav, M.Sabitha \& C.J.Sampath Kumar

Lactonova Nutripharm (P) Ltd, Makers of ASTASHINE Capsules, 81/3, IDA Mallapur, Hyderabad, Telangana, India-500 076.

DOI: 10.38177/AJBSR.2020.2303

\section{ABSTRACT}

Astaxanthin is a naturally occurring carotenoid which is derived from the microalgae Haematococcuspluvialis. As well as being the most powerful antioxidant known to science, it also has potent anti-inflammatory properties. Naturalastaxanthin's distinct advantage in comparison to other antioxidants, is its ability to span the entire lipid bilayer of the cell membrane, thus providing superior protection from the inside out. Natural astaxanthin has a strong ability to both balance and strengthen the immune system. This article reviews the current available scientific literature regarding the effect of astaxanthin from the algae Haematoccus pluvialis in Astashine capsules as a natural immune booster in covid-19 infections. Keywords: Astashine capsules, Immunity, Natural immune booster, Covid-19.

\section{Introduction}

The recent emergence of the SARS-CoV-2 viral pandemic has underscored the importance of maintaining pulmonary health as well as the need to minimize SARS-CoV-2 induced chronic inflammation and the resulting pathologic levels of inflammatory cytokines. SARS-CoV-2 infection results in COVID-19 syndrome that includes coughing, sneezing, fever, pneumonia, and potentially death.

Current treatments for viral infection and the ensuing chronic inflammation include anti-viral medications as well as steroidal and non-steroidal anti-inflammatories (NSAIDS); however, safety and efficacy limitations restrict anti-inflammatory treatment regimens for many patients. Coronaviruses have been shown to induce lung damage by increasing inflammatory signaling pathways and cytokine production leading to elevated immune cell infiltration and macrophagic polarization shifts (M2 to M1). Astaxanthin (ASTAXANTHIN), a safe, orally bioavailable, naturally occurring molecule with strong anti-inflammatory and antioxidant activity, has been shown to (i) significantly attenuate pathological elevation of critical inflammatory cell signaling pathways (NF-kB), (ii) decrease the resulting elevated proinflammatory cytokine levels, (iii) reduce immune cell infiltration of the lung, and (iv) positively influence macrophage polarization in humans and animal models of disease.

Corona represents crown-like spikes on the outer surface of the virus thus, it was named as a coronavirus. Coronaviruses are minute in size (65-125 $\mathrm{nm}$ in diameter) and contain a single-stranded RNA as a nucleic material, size ranging from 26 to 32kbs in length. The severe acute respiratory syndrome coronavirus (SARS-CoV), H5N1 influenza A, H1N1 2009 and Middle East respiratory syndrome coronavirus (MERS-CoV) cause acute lung injury (ALI) and acute respiratory distress syndrome (ARDS) which leads to pulmonary failure and result in fatality. These viruses were thought to infect only animals until the world witnessed a severe acute respiratory syndrome (SARS) outbreak caused by SARS-CoV, in China.

This virus was reported to be a member of the $\beta$ group of coronaviruses. The novel virus was named as Wuhan coronavirus or 2019 novel coronavirus (2019-nCov) by the Chinese researchers. 


\section{History}

There are various theories about where the very first case may have originated. The first known case may trace back to 1 December 2019 in Wuhan Hubei, China. Within a month, the number of coronavirus cases in Hubei gradually increased. According to official Chinese sources these were mostly linked to the Huanan sea food market, which also sold live animals, and one theory is that the virus came from one of these animals.

A pneumonia cluster of unknown cause was observed on 26 December and treated by the doctor in Hubei Provincial Hospital, On 30 December, a group of doctors at Wuhan Central Hospital alerted their colleagues of a "SARS-like coronavirus".

During the early stages of the outbreak, the number of cases doubled approximately every seven and a half days. In early and mid-January 2020, the virus spread to other Chinese provinces helped by the Chinese New Year migration and Wuhan being a transport hub and major rail interchange. On 20 January, China reported nearly 140 new cases in one day, including two people in Beijing and one in Shenzhen Later official data shows 6,174 people had already developed symptoms by then, and more may have been infected. A report in the lancet on 24 January indicated human transmission, strongly recommended personal protective equipment for health workers, and said testing for the virus was essential due to its "pandemic potential". On 30 January, the WHO declared that the coronavirus was a public health emergency for international concern

By 26 March, the United States had overtaken China and Italy with the highest number of confirmed cases in the world corona virus genome indicates the majority of COVID-19 cases in New York came from European travellers, rather than directly from China or any other Asian country.

\section{Origin}

Based on virus genome sequencing results and evolutionary analysis, bat has been suspected as natural host of virus origin, and SARS-CoV-2 might be transmitted from bats via unknown intermediate hosts to infect humans. It is clear now that SARS-CoV-2 could use angiotensin-converting enzyme 2 (ACE2), the same receptor as SARS-CoV to infect humans. Several studies suggested that bat may be the potential reservoir of SARS-CoV-2 suggesting that bat CoV and human

\section{Epidemiology}

Data provided by the WHO Health Emergency Dashboard (March 14, 06.00 am CET) report 142.320 confirmed cases worldwide since the beginning of the epidemic. 5.388 (3.78\%) cases have been fatal.

In china, 81.021 (57\%) cases confirmed clinically and, in the laboratory, and 3.173 deaths are reported. In addition to China, there are 61.299 confirmed cases in 129 other countries. The countries with most cases are Italy (17.660) and the Islamic Republic of Iran (11.364). The epidemiological scenario, therefore, has drastically changed, as on March 3 about $92 \%$ (79.968) of the confirmed cases were recorded in China, where almost all the deaths were also recorded $(2,873,96.5 \%)$. Of note, the "confirmed" cases reported between February 13, 2020, and February 19, 2020, include both laboratory-confirmed and clinically diagnosed patients from the Hubei province. 
The most up-to-date source for the epidemiology of this emerging pandemic can be found at the following sources:

- The WHO Novel Coronavirus (COVID-19) Situation Board

- The Johns Hopkins Centre for Systems Science and Engineering site for Coronavirus Global Cases COVID-19, which uses openly public sources to track the spread of the epidemic.

\section{Sex differences}

The impact of the pandemic and its mortality rate are different for men and women. Mortality is higher in men in studies conducted in China and Italy. The highest risk for men is in their 50s, with the gap between men and women closing only at 90 . In China, the death rate was 2.8 percent for men and 1.7 percent for women. The exact reasons for this sex-difference are not known, but genetic and behavioural factors could be a reason. Sex-based immunological differences, a lower prevalence of smoking in women, and men developing co-morbid conditions such as hypertension at a younger age than women could have contributed to the higher mortality in men. In Europe, of those infected with COVID-19, 57\% were men; of those infected with COVID-19 who also died, 72\% were men. As of April 2020, the U.S. government is not tracking sex-related data of COVID-19 infections. Research has shown that viral illnesses like Ebola, HIV, influence, and SARS affect men and women differently. A higher percentage of health workers, particularly nurses, are women, and they have a higher chance of being exposed to the virus.

\section{Pathogenesis}

ACE2, found in the lower respiratory tract of humans, is known as cell receptor for SARS-CoV and regulates both the cross-species and human-to-human transmission. The virion S-glycoprotein on the surface of coronavirus can attach to the receptor, ACE2 on the surface of human cells. S glycoprotein includes two subunits, S1 and S2. S1 determines the virus-host range and cellular tropism with the key function domain-RBD, while S2 mediates virus-cell membrane fusion by two tandem domains, heptad repeats (HR1) and HR2. After membrane fusion, the viral genome RNA is released into the cytoplasm, and the uncoated RNA translates two polyproteins, pp1a and pplab, which encode non-structural proteins, and form replication-transcription complex (RTC) in double-membrane vesicle .Lastly, the virion-containing vesicles fuse with the plasma membrane to release the virus.

\section{Signs and Symptoms}

The most common symptoms of COVID-19 are fever, dry cough, and tiredness. Some patients may have aches and pains, nasal congestion, sore throat or diarrhoea. These symptoms are usually mild and begin gradually. Some people become infected but only have very mild symptoms. Most people (about 80\%) recover from the disease without needing hospital treatment. Around 1 out of every 5 people who gets COVID-19 becomes seriously ill and develops difficulty breathing. Older people, and those with underlying medical problems like high blood pressure, heart and lung problems, diabetes, or cancer, are at higher risk of developing serious illness. However, anyone can catch COVID-19 and become seriously ill. Even people with very mild symptoms of COVID-19 can transmit the virus. People of all ages who experience fever, cough and difficulty breathing should seek medical attention. 


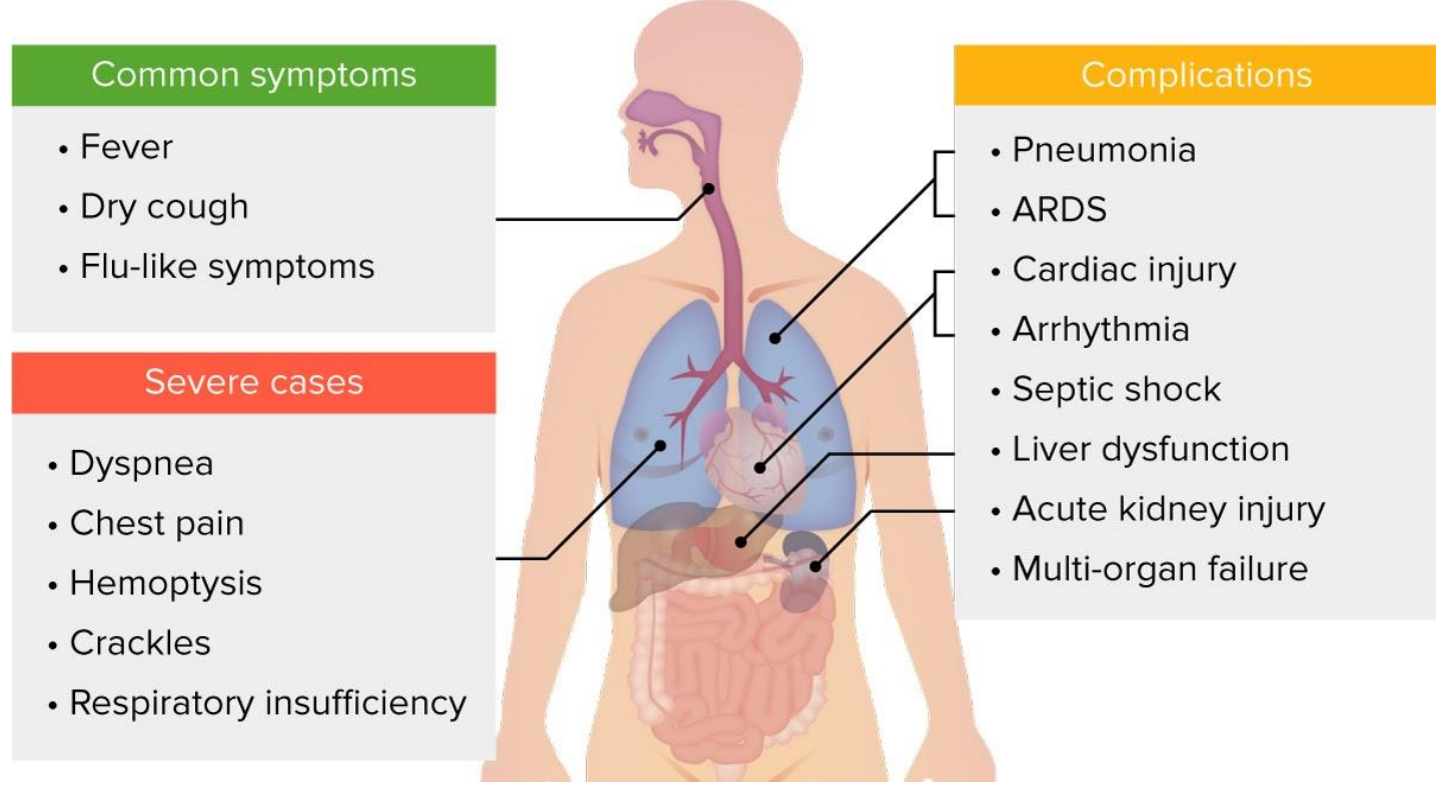

\section{Transmission}

People can catch COVID-19 from others who have the virus. The disease spreads primarily from person to person through small droplets from the nose or mouth, which are expelled when a person with COVID-19 coughs, sneezes, or speaks. These droplets are relatively heavy, do not travel far and quickly sink to the ground. People can catch COVID-19 if they breathe in these droplets from a person infected with the virus. This is why it is important to stay at least 1 metre (3 feet) away from others. These droplets can land on objects and surfaces around the person such as tables, doorknobs and handrails. People can become infected by touching these objects or surfaces, then touching their eyes, nose or mouth. This is why it is important to wash your hands regularly with soap and water or clean with alcohol-based hand rub.

\section{Immunopathology}

Although SARS-COV-2 has a tropism for ACE2-expressing epithelial cells of the respiratory tract, patients with severe COVID-19 have symptoms of systemic hyper inflammation. Clinical laboratory findings of elevated IL-2, IL-7, IL-6, granulocyte macrophage colony stimulating factor (GM-CSF), interferon inducible protein (IP-10), monocyte chemoattractant protein (MCP-1), macrophage inflammatory protein (MIP-1 $\alpha$ ), and tumour necrosis factor (TNF- $\alpha$ ) indicative of (CRS) suggest an underlying immunopathology.

Additionally, people with COVID-19 and acute respiratory distress syndrome (ARDS) have classical serum biomarkers of CRS, including elevated c-reactive protein (CRP), lactate dehydrogenase (LDH), D-dimer, and ferritin

\section{Virology}

Severe acute respiratory syndrome coronavirus 2 (SARS-CoV-2) is a novel virus, first isolated from three people with pneumonia connected to the cluster of acute respiratory illness cases in Wuhan. All features of the novel SARS-CoV-2 virus occur in related coronaviruses in nature. SARS-CoV-2 is closely related to SARS-CoV, and is thought to have a zoonotic origin. SARS-CoV-2 genetically clusters with the genus beta coronavirus, and is 96 per 
cent identical at the whole genome level to other bat coronavirus samples and 92 per cent identical to pangolin coronavirus

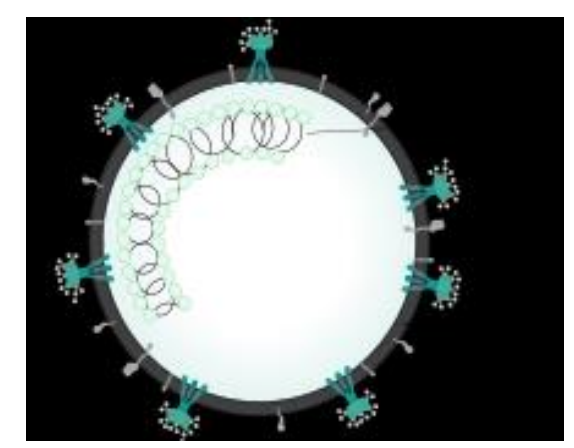

\section{Diagnosis}

\section{Viral testing}

The standard test for current infection with SARS-CoV-2 uses RNA testing of respiratory secretions collected using a nasopharyngeal swab, though it is possible to test other samples. This test uses real-time RT-PCR which detects presence of viral RNA fragments.

A number of laboratories and companies are developing serological tests, which detect antibodies produced by the body in response to infection.

\section{Diagnostic criteria}

The viral research institution in China has conducted preliminary identification of the SARS-CoV-2 through the classical Koch's postulates and observing its morphology through electron microscopy. So far, the golden clinical diagnosis method of COVID-19 is nucleic acid detection in the nasal and throat swab sampling or other respiratory tract samplings by real-time PCR and further confirmed by next-generation sequencing.

In laboratory examination results, most patients had normal or decreased white blood cell counts, and lymphocytopenia. But in the severe patients, the neutrophil count, D-dimer, blood urea, and creatinine levels were higher significantly, and the lymphocyte counts continued to decrease. Additionally, inflammatory factors (interleukin (IL)-6, IL-10, tumour necrosis factor- $\alpha(\mathrm{TNF}-\alpha)$ increase, indicating the immune status of patients. The data showed that ICU patients had higher plasma levels of IL-2, IL-7, IL-10, granulocyte colony-stimulating factor (GCSF), $10 \mathrm{kD}$ interferon-gamma-induced protein (IP-10), monocyte chemoattractant protein-1 (MCP-1), macrophage inflammatory protein 1- $\alpha$ (MIP-1 $\alpha)$, and TNF- $\alpha$.

\section{Treatment of COVID-19}

\section{Current therapies}

Antiviral drugs and systemic corticosteroid treatment commonly used in clinical practice previously, including neuraminidase inhibitors (oseltamivir, peramivir, zanamivir, etc.), ganciclovir, acyclovir, and ribavirin, as well as methylprednisolone for influenza virus, are invalid for COVID-19 and not recommended. Remdesivir has been reported to treat the first US case of COVID-19 successfully. Chloroquine is a repurposed drug with great potential 
to treat COVID-19. Chloroquine has been used to treat malaria for many years, with a mechanism that is not well understood against some viral infections. Chloroquine can inhibit $\mathrm{pH}$-dependent steps of the replication of several viruses with a potent effect on SARS-CoV infection and spread. Moreover, chloroquine has immunomodulatory effects, suppressing the production/release of TNF- $\alpha$ and IL-6. It also works as a novel class of autophagy inhibitor, which may interfere with viral infection and replication. A combination of remdesivir and chloroquine was proven to effectively inhibit the recently emerged SARS-CoV-2 in vitro.

\section{Antiviral therapy}

\section{Interferon-alpha (IFN $\alpha)$}

IFN $\alpha$ is a member of the family of type I IFNs that plays an important role in host resistance to viral infection. IFN $\alpha$ suppresses viral infection by directly interfering with replication of the virus and by promoting both innate and adaptive immune responses. In vitro experiments showed that IFN $\alpha$ effectively inhibits the replication of SARS-CoV. It has also been reported that cynomolgus monkeys are protected from infection with SARS CoV by treatment with IFN $\alpha$. Moreover, the therapeutic benefit of synthetic recombinant IFN $\alpha$ for patients with SARS was demonstrated in a pilot clinical trial. Thus, IFN $\alpha$ should be considered a candidate drug for COVID-19 therapy.

\section{Lopinavir/ritonavir (Kaletra)}

Lopinavir/ritonavir was first known as a protease inhibitor that interferes with the replication and synthesis of human immunodeficiency virus (HIV), leading to the production of immature, non-infectious virus particles. It has been reported that ritonavir and lopinavir can bind to the endopeptidase C 30 of SARS-CoV-2 protease as evaluated by molecular models. This suggests that lopinavir/ritonavir may exert an antiviral effect by inhibiting protein synthesis of SARS-CoV-2. In addition, several lines of evidence showed that treatment with lopinavir/ritonavir alone or in combination with other antiviral drugs was shown to improve the outcome of severe patients with SARS or MERS by ameliorating ARDS. SARS-CoV-2 is similar to these two viruses, may have a beneficial effect on COVID-19.

\section{Ribavirin}

Ribavirin is a nucleoside analogue with broad antiviral activity. It can prevent the replication of RNA and DNA viruses by suppressing the activity of inosine monophosphate dehydrogenase, which is required for the synthesis of guanosine triphosphate (GTP). Ribavirin was widely used to treat SARS patients with or without concomitant use of steroids during the outbreak of SARS in Hong Kong. Thus, ribavirin could be considered as a treatment option for COVID-19 patients.

\section{Chloroquine}

Chloroquine is a widely used antimalarial and autoimmune disease drug. Recently, chloroquine has been reported as a potential broad-spectrum antiviral drug. Research found that chloroquine effectively suppresses the recently emerged novel CoV (SARS-CoV-2) in vitro. Chloroquine is a cheap and safe drug that has been used for more than 70 years and thus it is potentially clinically applicable against COVID-19. 


\section{Arbidol (umifenovir)}

Arbidol is an antiviral drug against influenza infection that is widely used in Russia and China. Arbidol and arbidolmesylate were shown to have a potent inhibitory effect in reducing the reproduction of SARS-CoV in vitro. case reports and case series revealed that arbidol alone or combined with antiviral drugs produced certain benefits in the treatment of COVID-19 pneumonia. Currently, many randomised clinical controlled trials are being carried out

\section{Remdesivir}

The nucleoside analogue remdesivir (GS-5734) was reported to inhibit SARS-CoV and MERS-CoV in vivo. More recently, an in vitro study showed that remdesivir potently blocked SARS-CoV-2 infection at low-micromolar concentrations and showed a high selectivity index. In addition, the first case of SARS-CoV-2 infection in the USA was treated with intravenous remdesivir when the patient's condition deteriorated. Although remdesivir has some benefits for the treatment of COVID-19 pneumonia, randomised controlled trials are still required to determine its efficacy and safety.

Taken together, these antiviral drugs may be promising treatment options for the treatment of COVID-19. However, there are also a few points worth noting here:

$\checkmark$ The potential interaction of these antiviral drugs with other therapeutic drugs should be considered;

$\checkmark \quad$ Adverse reactions caused by lopinavir/ritonavir, such as diarrhoea, nausea, vomiting and liver damage, should be also considered;

$\checkmark \quad$ It is not recommended to use three or more antiviral drugs at the same time, and the use of related drugs should be stopped when there are intolerable side effects; and

$\checkmark \quad$ Further evaluation of the efficacy of current antiviral drugs in clinical applications is needed.

\section{Management}

People are managed with supportive care, which may include fluid therapy, oxygen support, and supporting other affected vital organs The CDC recommends that those who suspect they carry the virus wear a simple face mask. Extracorporeal membrane oxygenation(ECMO) has been used to address the issue of respiratory failure, but its benefits are still under consideration Personal hygiene and a healthy lifestyle and diet have been recommended to improve immunity Supportive treatments may be useful in those with mild symptoms at the early stage of infection.

The WHO, the Chinese national organisation, and the United States' national institute of health have published recommendations for taking care of people who are hospitalised with COVID-19.

\section{Prevention}

Preventive measures are the current strategy to limit the spread of cases. Because an epidemic will increase as long as $\mathrm{R} 0$ is greater than 1 (COVID-19 is 2.2), control measures must focus on reducing the value to less than 1 . 
Preventive strategies are focused on the isolation of patients and careful infection control, including appropriate measures to be adopted during the diagnosis and the provision of clinical care to an infected patient. For instance, droplet, contact, and airborne precautions should be adopted during specimen collection, and sputum induction should be avoided.

The WHO and other organizations have issued the following general recommendations:

- Avoid close contact with subjects suffering from acute respiratory infections.

- Wash your hands frequently, especially after contact with infected people or their environment.

- Avoid unprotected contact with farm or wild animals.

- People with symptoms of acute airway infection should keep their distance, cover coughs or sneezes with disposable tissues or clothes and wash their hands.

- Strengthen, in particular, in emergency medicine departments, the application of strict hygiene measures for the prevention and control of infections.

- Individuals that are immunocompromised should avoid public gatherings.

The most important strategy for the people to undertake is to frequently wash their hands and use portable hand sanitizer and avoid contact with their face and mouth after interacting with a possibly contaminated environment.

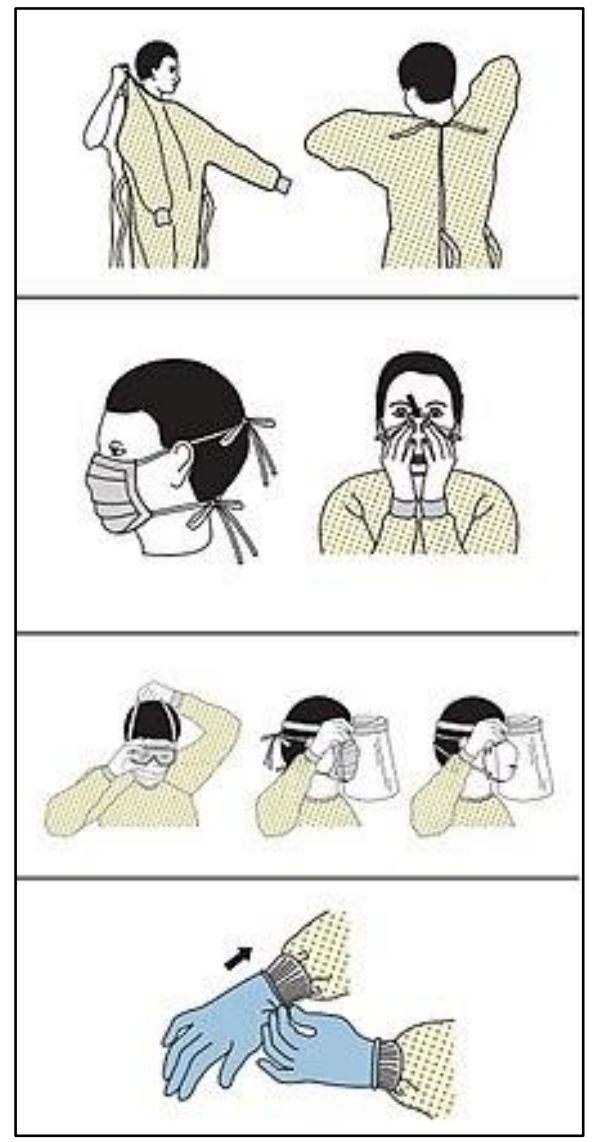

Healthcare workers caring for infected individuals should utilize contact and airborne precautions to include PPE such as N95 or FFP3 masks, eye protection, gowns, and gloves to prevent transmission of the pathogen.

Meanwhile, scientific research is growing to develop a coronavirus vaccine. In recent days, China has announced the first animal tests, and researchers from the University of Queensland in Australia have also announced that, after completing the three-week in vitro study, they are moving on to animal testing. Furthermore, in the U.S., the National Institute for Allergy and Infectious Diseases (NIAID) has announced that a phase 1 trial has begun for a novel coronavirus immunization.

The immune system is made up of a network of cells, tissues and organs that work together to protect the body against infectious microorganisms, such as certain bacteria and viruses; whilst also working to destroy any infectious microorganisms that manage to invade the body. Phagocytes are cells that destroy invading organisms, while lymphocytes are cells that allow the body to remember and recognize previous invaders and help the body destroy them - the innate immune response. Natural astaxanthin has shown positive effects and substantial benefit in enhancing the capacity of both lymphocytes and phagocytes. 


\section{Astashine capsules as a potent Immune Agent}

Inflammation is the body's biological response to harmful stimuli. Acute inflammation is the initial response to such stimuli and in most cases it can be visually identified. The other form of inflammation is "chronic inflammation"; it is often called the silent killer since there are very few, if any, symptoms of this type of inflammation. Unlike the acute type, chronic inflammation builds up in the body as a result of the immune system constantly responding to threats. Chronic inflammation is one of the major causes of accelerated aging and many of its associated diseases such as chronic heart disease, arthritis or allergies. It is characterized by the release of cytokines and pro-inflammatory markers such as tumor necrosis factor- $-\alpha$ (TNF- $-\alpha$ ),C-reactive protein (CRP) and others. Luckily, we can test for inflammation by measuring these different biological markers in the blood which will give an indication of inflammation levels. Natural astaxanthin has been shown to help significantly decrease the expression of pro-inflammatory markers and mediators, thus providing potent anti-inflammatory protection in the body. Several studies have been conducted to understand the anti-inflammatory mechanism of astaxanthin and it is thought to be related to the inhibition of the Nf-kB inflammatory pathway [5].

\section{Composition of Astashine capsules}

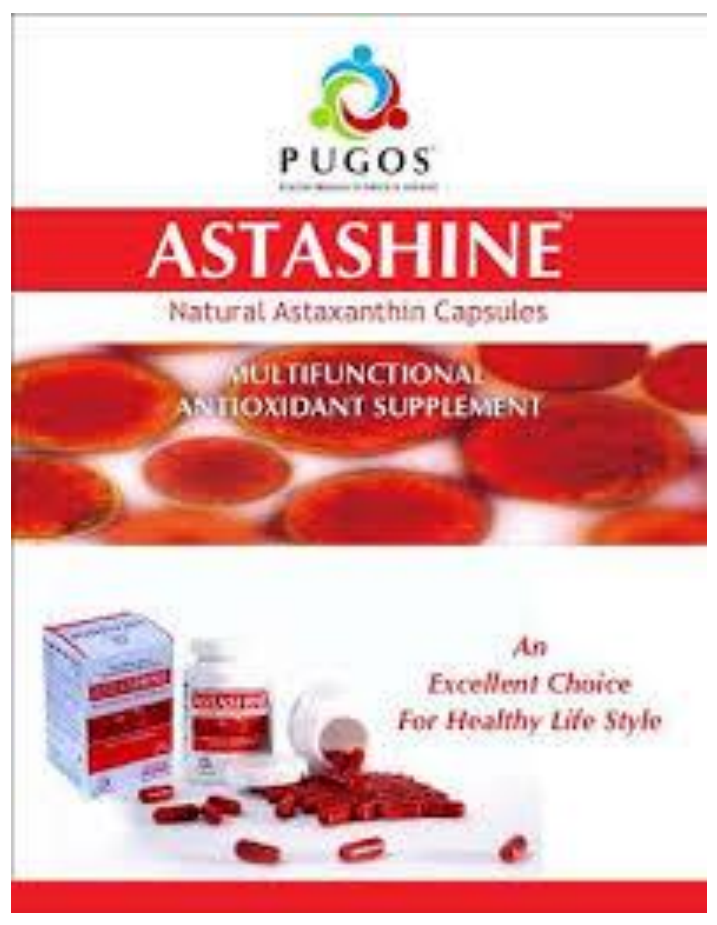

Astaxanthin - $2 m g$

(Naturally derived from Haematococcus pulvialis algae extract, which is microencapsulated)

\section{Mechanism of Action}

Astaxanthin's outstanding properties as an anti-inflammatory also play a major role in its ability to enhance immune function. Due to the multitude of ways in which Astaxanthin combats inflammation, it is a very special anti-inflammatory indeed. Both in-vitro and in-vivo research has been done to uncover its mechanism of action as an anti-inflammatory. This mechanism has been further demonstrated in several double blind, placebo controlled human clinical trials on various inflammatory conditions. 
Astaxanthin works to suppress different inflammatory mediators. Among these mediators are tumor necrosis factor alpha (TNF-a), prostaglandin E-2 (PGE-2), interleukin 1B (IL-1b) and nitric oxide (NO). In one experiment done with mice and also in-vitro, Astaxanthin was shown to suppress TNF-a, PGE-2, IL-1b, NO as well as the Cox-2 enzyme and nuclear factor kappa-B. [1-4]

Another study done the same year was led by a researcher from Japan's Hokkaido University Graduate School of Medicine. Here, the researchers found similar results: Astaxanthin was shown in vitro to decrease the production of NO, PGE-2 and TNF-a. This study also looked at Astaxanthin's anti-inflammatory effect in the eyes of rats. The researchers induced uveitis (inflammation of the inner eye including the iris) and found that Astaxanthin had a "dose dependent ocular anti-inflammatory effect, by the suppression of NO, PGE-2 and TNF-a production, through directly blocking nitric oxide synthase enzyme activity" [2]

\section{Clinical Study Reports of Astashine Capsules}

\section{Astashine capsules enhances Immune Response}

The immune system is made up of a network of cells, tissues and organs that work together to protect the body against infectious microorganisms, such as certain bacteria and viruses; whilst also working to destroy any infectious microorganisms that manage to invade the body.

Phagocytes are cells that destroy invading organisms, while lymphocytes are cells that allow the body to remember and recognize previous invaders and help the body destroy them - the innate immune response.

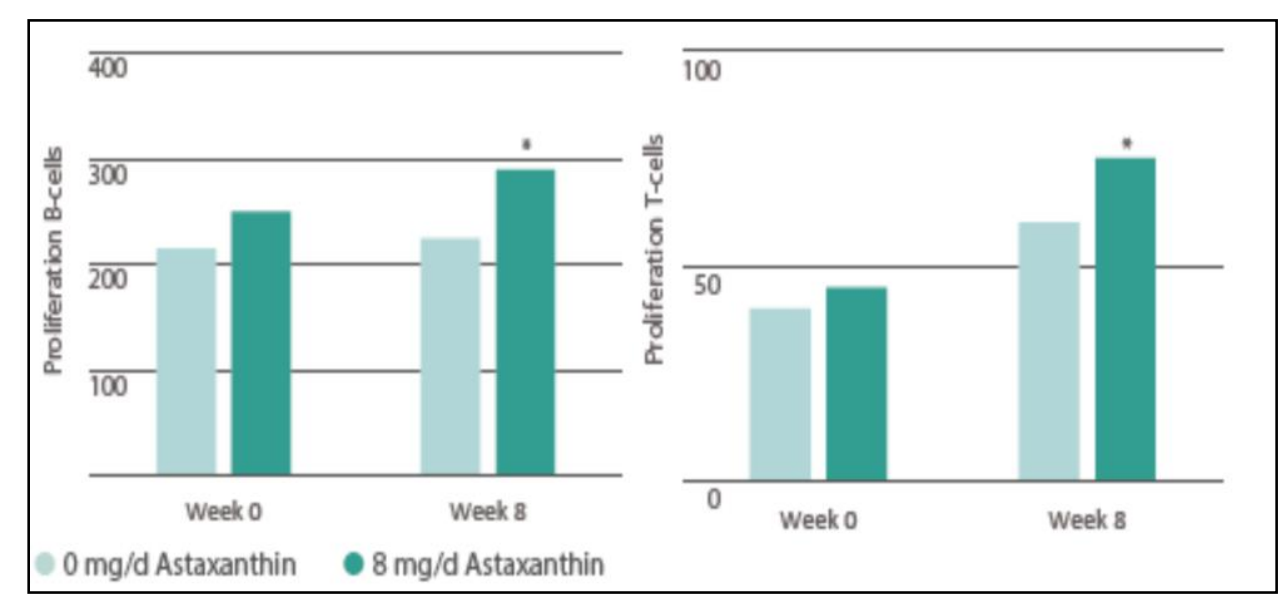

Figure 1: The effect of astaxanthin on immune response was measured in a double blind, randomized study involving 42 subjects. B-cells and T-cells were exposed to high concentrations of mitogens and their capacity to proliferate was measured $* \mathrm{p}<0.05$ compared with control.

Research has shown that natural astaxanthin is also a potent anti-inflammatory and is particularly effective against chronic inflammation. To test its efficacy, scientists have examined the levels of pro-inflammatory markers with and without the presence of astaxanthin. In a 2003 model study, Seonet al. found that astaxanthin significantly decreased the expression of different pro-inflammatory markers in Lipopolysaccharide-induced inflammation (LPS). LPS=Lipopolysaccharide AX=Astaxanthin 

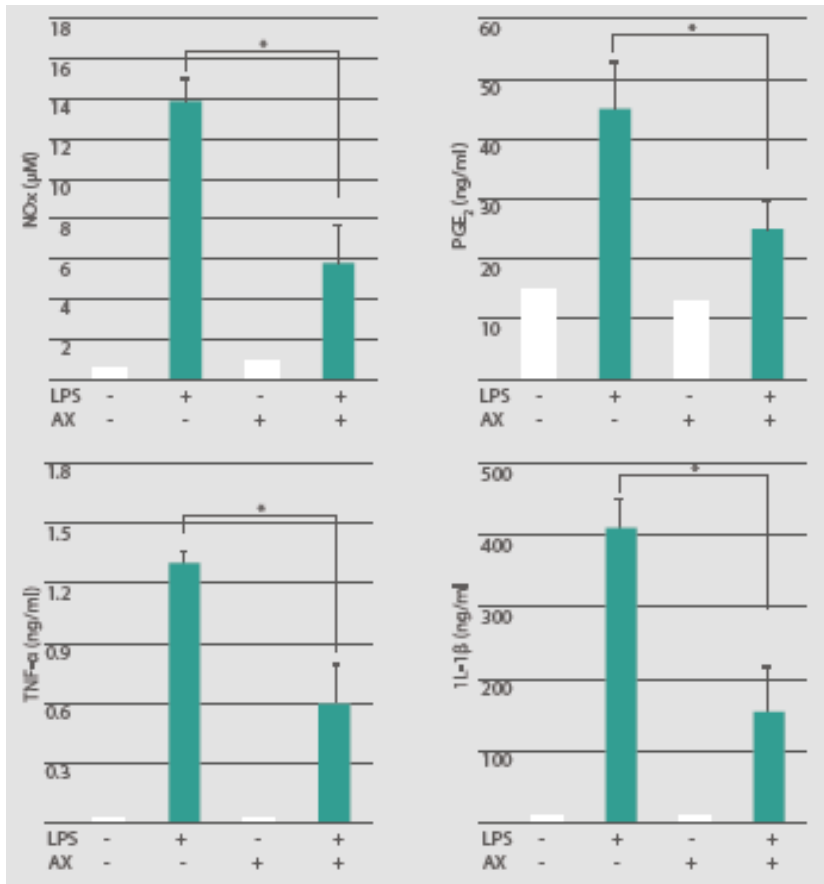

Figure 2: LPS-induced inflammation showing significant decreases in plasma levels of pro-inflammatory markers NO, PGE2, TNF- $\alpha$, and IL- $1 \beta$

In another randomized, double blind placebo controlled study, Park et al. (2010) examined 42 subjects for the pro-inflammatory marker CRP. The astaxanthin group supplemented with $2 \mathrm{mg} / \mathrm{d}$ for 8 weeks showed that the levels of CRP were significantly reduced. [2]

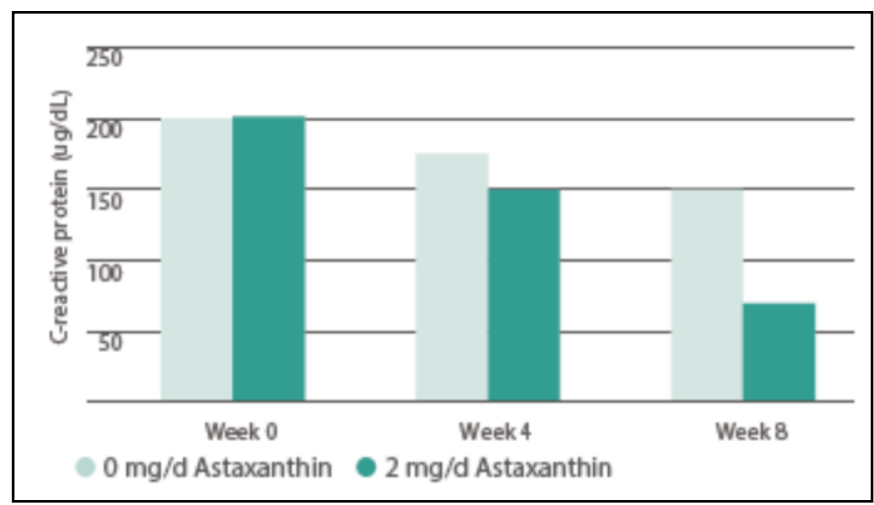

Figure 3: 8 weeks of supplementation with $2 \mathrm{mg} / \mathrm{d}$ natural astaxanthin significantly reduced the expression of the pro-inflammatory marker C-reactive protein (CRP).

\section{Safety of Astashine Capsules}

Astaxanthin has demonstrated safety in numerous human clinical trials. In one open-label clinical study on subjects with metabolic syndrome $(\mathrm{n}=17)$. Astaxanthin $(16 \mathrm{mg} /$ day, for three months) significantly raised blood bilirubin $(p \leq 0.05)$, potassium $(p \leq 0.05)$, and creatine kinase $(p \leq 0.01)$, although all three values remained within normal range. Also, astaxanthin significantly lowered the liver enzyme gamma-glutamyl transpeptidase (GGTP; $\mathrm{p} \leq 0.05$ ). Since the researchers noted this enzyme was abnormally elevated in 11 of the 17 subjects at baseline, this astaxanthin effect may have been beneficial. Animal experiments have investigated astaxanthin at levels well over 
$120 \mathrm{mg} /$ day in human equivalents, without causing apparent harm. Hoffman-La Roche confirmed its safety with extensive tests, including acute toxicity, mutagenicity, teratogenicity, embryotoxicity, and reproductive toxicity.

\section{Suggested Dosage}

The doses of astaxanthin used in clinical trials have ranged from $1 \mathrm{mg} /$ day to $40 \mathrm{mg} / \mathrm{day}$ (with the majority in the 6-12 mg range); single-dose pharmacokinetic studies used up to $100 \mathrm{mg}$ per dose. As a dietary supplement, astaxanthin should be taken along with fats, with or immediately prior to meals, to ensure its optimal absorption.

\section{Summary \& Conclusion}

The novel coronavirus originated from the Hunan seafood market at Wuhan, China where bats, snakes, raccoon dogs, palm civets, and other animals are sold, and rapidly spread up to 109 countries. The zoonotic source of SARS-CoV-2 is not confirmed, however, sequence-based analysis suggested bats as the key reservoir. DNA recombination was found to be involved at spike glycoprotein which assorted SARS-CoV (CoVZXC21 or CoVZC45) with the RBD of another Beta CoV, thus could be the reason for cross-species transmission and rapid infection. According to phylogenetic trees, SARS-CoV is closer to SARS-like bat CoVs. Until now, no promising clinical treatments or prevention strategies have been developed against human coronaviruses. However, the researchers are working to develop efficient therapeutic strategies to cope with the novel coronaviruses. Various broad-spectrum antivirals previously used against influenza, SARS and MERS coronaviruses have been evaluated either alone or in combinations to treat COVID-19 patients, mice models, and clinical isolates. Remdesivir, Lopinavir, Ritonavir, and Oseltamivir significantly blocked the COVID-19 infection in infected patients. It can be concluded that the homologous recombination event at the S protein of RBD region enhanced the transmission ability of the virus. While the decision of bring back the nationals from infected area by various countries and poor screening of passengers, become the leading cause of spreading virus in others countries.

Most importantly, human coronaviruses targeting vaccines and antiviral drugs should be designed that could be used against the current as well as future epidemics. There are many companies working for the development of effective SARS-CoV-2 vaccines, such as Modern Therapeutics, Innova Pharmaceuticals, Novavax, Vir Biotechnology, Stermirna Therapeutics, Johnson \& Johnson, VIDO-InterVac, GeoVax-BravoVax, Clover Biopharmaceuticals, CureVac, and Codagenix. But there is a need for rapid human and animal-based trails as these vaccines still require 3-10 months for commercialization. There must be a complete ban on utilizing wild animals and birds as a source of food. Beside the development of most efficient drug, a strategy to rapidly diagnose SARS-CoV-2 in suspected patient is also required. The signs and symptoms of SARS-CoV-2 induced COVID-19 are a bit similar to influenza and seasonal allergies (pollen allergies). Person suffering from influenza or seasonal allergy may also exhibit temperature which can be detected by thermo-scanners; hence the person will become suspected. Therefore, an accurate and rapid diagnostic kit or meter for detection of SARS-CoV-2 in suspected patients is required, as the PCR based testing is expensive and time consuming. Different teams of Chinese doctors should immediately sent to European and other countries, especially Spain and Italy to control the over spread of COVID-19, because Chinese doctors have efficiently controlled the outbreak in china and limited the mortality rate to less than $3 \%$ only. The therapeutic strategies used by Chinese, should also be followed by other countries. 
The SARS-CoV-2 virus (Severe Acute Respiratory Syndrome Coronavirus 2) recently emerged in China and has rapidly spread worldwide leading the World Health Organization to classify it as a pandemic. SARS-CoV-2 is closely related (70\% genetically identical) to the SARS-CoV-1 virus that was responsible for a total of 774 deaths in 17 different countries in 2002-2003. Both viruses belong to the Coronaviridae "crown" family of enveloped, single-stranded positive-sense RNA genome viruses and both pandemics resulted from zoonotic transmission (animal reservoirs to human hosts). SARS-CoV-2 induced syndrome, referred to as Coronavirus Disease 2019 (COVID-19), consists of coughing, sneezing, fever, pneumonia and potentially pulmonary failure/death. The currently available pharmaceutical treatments are very limited and include anti-virals (remdesivir, ritonavir, lopinavir). Due to the complexity of anti-inflammation strategies to reduce virus-associated pathology versus attenuation of immune-mediated viral clearance, utilization of steroids and NSAIDSs (ibuprofen) are questionable. Potential treatments under development include prophylactic vaccines, chloroquine, angiotensin II receptor type I inhibitors (losartan, candesartan) and various targeted anti-inflammatories including Actemra (tocilizumab, IL-6 inhibitor). While it is too early to extensively discover the exact or unique pathomechanisms involved in SARS-CoV-2 infection/disease, observations of COVID-19 patient physiology as well as genetic similarities between the two viruses, support the assumption that SARS-CoV-2 molecular mechanisms will be similar to those of SARS-CoV-1. Both viruses infect the lungs and gastrointestinal tract and appear to utilize a membrane incorporated "spike" glycoprotein interaction with human angiotensin-converting enzyme 2 (ACE2) for cell attachment/entry. Pathophysiologically, SARS-CoV-1 infection results in upregulation of NF-kB inflammatory signaling pathway, increased proinflammatory cyokine levels (TNF-a, IL-6, COX-2, etc.), enhanced immune cell infiltration into the lung (due in part to increased levels of Monocyte Chemoattractant Protein-1 (MCP-1)), and a heightened level of macrophagic polarity shift from the healing M2 phenotype towards the proinflammatory M1 phenotype. These inflammatory mechanisms lead to acute lung injury, pneumonia, and ultimately pulmonary failure. Indeed, several studies have underscored the capacity for SARS-CoV-1 viral $\mathrm{N}$ and Spike proteins to specifically upregulate NF-kB gene expression. Additionally, mice infected with a genetically modified SARS-CoV-1 virus lacking the ability to upregulate NF-kB demonstrate increased survival, decreased immune cell infiltration in lung and reduced inflammatory cytokine levels (TNF-a, IL-6). Application of NFkB inhibitors in this mouse model also increased survival following unmodified SARS-CoV-1 infection and reduced inflammation and lung pathology. SARS-CoV-1 activation of the NF-kB pathway results in elevated proinflammatory cytokines levels (TNF-a, IL-6, MCP-1, etc.). Additionally, pathogen-associated molecular patterns activate the "inflammasome" further upregulating inflammatory cyokines levels (IL-1b, IL-18). SARS-CoV-1 proteins have also been shown to upregulate cyclooxygenase-2 (COX-2). Importantly, prolonged elevation of these cytokines leads to acute lung injury. Studies on SARS-CoV-1 infection have shown an important shift in macrophage polarization from the healing M2 phenotype (produces IL-4 and IL-10) to the proinflammatory M1 phenotype (produces inflammatory cytokines IL-1b, IL-6, TNF-a). In addition, both increased NF-kB inflammatory signaling and increased M1 macrophages elevate MCP-1 levels contributing to increased infiltration of immune cells (mast cells, neutrophils, macrophages, etc.) into the lungs further exacerbating the proinflammatory environment and ultimately leading to lung complications. Astaxanthin helps to maintain lung health and may minimize 
SARS-CoV-2 effects Astaxanthin (ASTAXANTHIN) is a safe, orally bioavailable, naturally occurring molecule with strong antioxidant and anti-inflammatory activity. Following oral administration and intestinal uptake, ASTAXANTHIN is delivered initially to the liver via chylomicrons and subsequently distributed systemically to tissues throughout the body integrating within cellular and mitochondrial membranes. ASTAXANTHIN completely traverses the lipid bilayer component of cell membranes, facilitating its anti-oxidant functions. Mitochondrial ASTAXANTHIN localization allows for the unique regulation of redox stress at the source. Astaxanthin has been shown in humans to significantly lower important inflammatory and metabolic disease measures including tumor necrosis factor-alpha (TNF-a), low density lipoprotein cholesterol (LDL-C), apolipoprotein B (ApoB), and triglycerides, while significantly raising adiponectin and high density lipoprotein cholesterol (HDL-C) levels. 10-13 ASTAXANTHIN has also positively affected markers of oxidative stress in obese humans including significantly lowering isoprostanes and malondialdehyde (MDA) levels and significantly increasing total antioxidant capacity and superoxide dismutase (SOD) Importantly to lung inflammation and SARS-CoV2 infection, ASTAXANTHIN has been shown to significantly decrease NF-kB pathway signaling both in vitro and in vivo. Reduction of NF-kB signaling leads to decreased levels of the inflammatory cytokines seen elevated in SARS-CoV-1 infections. Indeed, ASTAXANTHIN has been shown to, at very low doses, reduce TNF-a in humans. In rodents, ASTAXANTHIN reduces TNF-a equivalent to a corticosteroid, the gold standard of anti-inflammatory compounds, with no evidence of immunosuppressive effects. ASTAXANTHIN also significantly decreased other important mediators of inflammation in animal models including IL-6, IL-1b, COX-2, CRP, PGE-2, iNOS, and nitric oxide (NO). ASTAXANTHIN has also been shown to reduce inflammatory cell infiltration (neutrophils, eosinophils, macrophages) in a lung model of inflammation. In fact, the reduction response measured with ASTAXANTHIN was superior to Ibuprofen treatment. This is further supported by observations of MCP-1 reduction following ASTAXANTHIN treatment that will in turn lead to inflammatory cell infiltration attenuation. In addition to reduced macrophagic infiltration, ASTAXANTHIN has been shown to reduce the numbers of M1 macrophages displaying M1 phenotype in favor of M2 phenotype. In addition to inhibition of NF-kB pathway activation, reduction in the M1/M2 macrophage phenotype ratio is important in decreasing levels of inflammatory cytokines.

ASTAXANTHIN is well suited for chronic administration. Conclusion In summary, ASTAXANTHIN has the potential, as a safe and strong anti-inflammatory molecule, to maintain healthy lung function as well as the potential to decrease SARS-CoV-2 associated pulmonary pathophysiology by (i) reducing NF-kB pathway activation, (ii) decreasing inflammatory cytokine production, (iii) attenuating immune cell infiltration, and (iv) shifting macrophage population phenotype (M1 to M2). Studies demonstrate that astaxanthin helps to balance the immune system by stimulating its infection, while also helping suppress the overactive immune responses that create needless inflammation. [6]

Astaxanthin increases the numbers and activity of white blood cells called lymphocytes and natural killer cells that are responsible for creating the body's innate immune response to invaders. [7-9] 
Asian Journal of Basic Science \& Research Volume 2, Issue 3, Pages 28-42, July-September 2020

Astaxanthin has similar immune-boosting effects in humans, improving the ability of protective white blood cells to surround and destroy infecting organisms, especially fungi such asCandida albicans. [10] Astaxanthin also protects human lymphocytes and neutrophils against the oxidant stresses imposed by the actions of certain white blood cells without reducing the killing effects of white blood cells themselves. [11]

Human studies reveal astaxanthin's beneficial actions on immune system in patients with allergies and asthma. When astaxanthin was given to asthmatic patients, it suppressed reactive cell activation as well as or better than the antihistamine drugs.[12]

\section{References}

1. Chew, B.P. et al., 2011. Dietary astaxanthin enhances immune response in dogs. Vet lmmunol and lmmunopathol.140 (2011) 199-206.

2. Park, J.S., Chyun, J.H., Kim, Y.K., Line, LL., Chew, B.P., 2010.Astaxanthin decreased oxidative stress and inflammation and enhanced immune response in humans. Nutr. Metab.5, 7-18.

3. Park, J.S., Kim, H.W., Mathison, B.D., Hayek, M.G., Massimino, S., Reinhart, G.A., Chew, B.P., $2010 b$. Astaxanthin uptake in domestic dogs and cats. Nutr. Metab.7, 52-59.

4. Macedo RC et al., Astaxanthi $\mathrm{n}$ addition improves humanneutrophils function: in vitro study.Eur $\mathrm{J}$ Nutr.2010;49:447- 457.

5. Seon-Jin L. et al. 2003. Astaxanthin Inhibits Nitric Oxide Production and Inflammatory Gene Expression by Suppressing IкB Kinase-dependent NF-кB Activation. Mol. Cells, Vol. 16, No. 1, pp. 97-105.

6. Chew BP, Park JS. Carotenoid action on the immune response. J Nutr. 2004 Jan;134(1):257S-61S.

7. Nakao R, Nelson OL, Park JS, Mathison BD, Thompson PA, Chew BP. Effect of dietary astaxanthin at different stages of mammary tumor initiation in BALB/c mice. Anticancer Res. 2010 Jun;30(6):2171-5.

8. Chew BP, Mathison BD, Hayek MG, Massimino S, Reinhart GA, Park JS. Dietary astaxanthin enhances immune response in dogs. Vet Immunol Immunopathol. 2011 Apr 15;140(3-4):199-206.

9. Park JS, Mathison BD, Hayek MG, Massimino S, Reinhart GA, Chew BP. Astaxanthin stimulates cell-mediated and humoral immune responses in cats. Vet Immunol Immunopathol. 2011 Dec 15;144(3-4):455-61.

10. Macedo RC, Bolin AP, Marin DP, Otton R. Astaxanthin addition improves human neutrophils function: in vitro study. Eur J Nutr. 2010 Dec;49(8):447-57.

11. Bolin AP, Guerra BA, Nascimento SJ, Otton R. Changes in lymphocyte oxidant/antioxidant parameters after carbonyl and antioxidant exposure. Int Immunopharmacol. 2012 Dec;14(4): 690-7.

12. Mahmoud FF, Haines DD, Abul HT, Abal AT, Onadeko BO, Wise JA. In vitro effects of astaxanthin combined with ginkgolide B on T lymphocyte activation in peripheral blood mononuclear cells from asthmatic subjects. $J$ Pharmacol Sci. 2004 Feb;94(2):129-36. 